\title{
Meeting report on an NHLBI workshop on ex vivo expansion of stem cells, July 29, 1999, Washington, D.C.
}

\author{
Catherine M. Verfaillie \\ Stem Cell Institute, Cancer Center and Department of Medicine, University of Minnesota, Minneapolis, Minn., USA
}

(Received 21 December 1999; accepted 28 December 1999)

\section{Organization and goals of conference}

A workshop organized by the National Heart Lung and Blood Institute (NHLBI) was held on ex vivo expansion of hematopoietic stem cells (HSC) at the National Institute of Health (NIH) in Bethesda on July 29, 1999. The meeting was organized by Leann Jensen, Ph.D., NHLBI, Bethesda, Maryland, in collaboration with Cynthia Dunbar, M.D., Hematology Branch, NHLBI, Bethesda, Maryland; Catherine Verfaillie, M.D., University of Minnesota, Minneapolis, Minnesota; and John Wagner, M.D., University of Minnesota, Minneapolis, Minnesota. The speakers included: Steve Bartelemez, Ph.D., Seattle Biomedical Research Institute, Seattle, Washington; Stephen Emerson, M.D., Ph.D., University of Pennsylvania, Philadelphia, Pennsylvania; Shelly Heimfeld, Ph.D., Fred Hutchinson Cancer Research Center, Seattle, Washington; Ihor Lemischka, Ph.D., Princeton University, Princeton, New Jersey; Ian McNiece, Ph.D., University of Colorado Health Sciences Center, Denver, Colorado; Kateri Moore, DVM, Princeton University, Princeton, New Jersey; Malcolm Moore, Ph.D., Memorial Sloan Kettering Cancer Center, New York, New York; Jan Nolta, Ph.D., Children's Hospital of Los Angeles, Los Angeles, California; Peter Quesenberry, M.D., University of Massachusetts Cancer Center, Worcester, Massachusetts; Dr. Richard Stephens, Chief, Oncology Branch, FDA, Rockville, Maryland; Beverly TorokStorb, Ph.D., Fred Hutchinson Cancer Research Center, Seattle, Washington; and Esmail Zanjani, Ph.D., VA Medical Center, Reno, Nevada.

The goal of the workshop was to identify important, unresolved questions in the area of ex vivo expansion of $\mathrm{HSC}$, with a special emphasis on umbilical cord blood (UCB) grafts. Questions addressed included which assays (in vivo transplantation models or in vitro assays) can measure human (non-human-primate) HSC? What is the genetic characterization of long-term repopulating HSC? How is "engraftment" affected by "homing" of human HSC to the bone marrow (BM) microenvironment? What are the requirements of culture conditions that support ex vivo HSC ex-

Offprint requests to: Catherine M. Verfaillie, M.D., Professor of Medicine, University of Minnesota, Box 806 UMHC, 422 Delaware Street SE, Minneapolis, MN 55455 USA; E-mail: verfa001@tc.umn.edu pansion? What cells do we need to expand: HSC, or committed myeloid and/or lymphoid cells? Finally, what are the regulatory issues that pertain to ex vivo expanded HSC?

\section{Presently utilized functional stem cell assay systems}

An initial topic that was addressed was the question of which assay system(s) is best suited to measure human HSC. Several speakers addressed the ability of in vivo transplantation models to measure engrafting cells, while others described in vitro assays that measure primitive progenitors. Drs. Nolta, Zanjani, and Dunbar described three different animal models that allow evaluation of engrafting human (non-human-primate) HSC in vivo, namely the Beige-Nude Severe Combined Immunodeficiency (SCID) (BNX) mouse, pre-immune fetal sheep, and RhesusMacaque transplantation models. The three investigators provided evidence for the validity of the model by testing engraftment of unmanipulated HSC populations as well as ex vivo manipulated HSC. Using retrovirally marked cells, Dr. Nolta showed that the BNX model can assess presence of multilineage repopulating cells that differentiate into lymphoid as well as myeloid progeny, as progeny contains the retroviral gene in the same insertion location [1]. She provided evidence that ex vivo expansion of $\mathrm{CD}^{3} 4^{1}$ cells from adult BM, but not UCB, interferes with multilineage differentiation following transplantation as few, if any, B cells were generated in BNX mice.

Like Dr. Nolta, Dr. Dunbar uses retroviral marking of stem cells to demonstrate multilineage differentiation of single transplanted HSC in vivo. She uses the non-human-primate RhesusMacaque transplantation model to address the question of stem cell engraftment [2]. Consistent with data presented by Drs. Nolta and Zanjani, the non-human-primate transplantation model demonstrated that engraftment of ex vivo expanded HSC was significantly poorer than had been encountered with cells that had simply been retrovirally transduced.

Dr. Zanjani provided evidence that the fetal sheep transplantation model allows characterization of short-term and long-term engrafting HSC [3]. He described two methods to determine the longevity of HSC: one method is to serially transfer human cells from primary to secondary and tertiary 
sheep recipients; a second method is to allow fetal sheep to be born, and give the animals multiple rounds of high dose human cytokines. In both systems, exhaustion of human hematopoiesis was observed for cell populations that may contain only short-term, but not long-term, engrafting HSC. This was exemplified by lack of exhaustion of $\mathrm{CD} 34^{1} \mathrm{CD} 38^{2}$ but not $\mathrm{CD} 34^{1} \mathrm{CD} 38^{1}$ adult $\mathrm{BM}$ cells, ex vivo expanded but not unmanipulated stem cells, CD $34^{1}$ versus $\mathrm{CD} 34^{2}$ cells from adult BM but not peripheral blood (PB).

Dr. Verfaillie described the use of an in vitro stroma-based assay to enumerate multilineage progenitors capable of undergoing self-renewing cell divisions [4]. This assay uses the AFT024 feeder discussed at length by Dr. K. Moore [5] during the meeting, on which single cells are sorted and their self-renewing and multilineage potential is examined for 8-14 weeks. Co-culture of CD34 ${ }^{1} \mathrm{Lin}^{2}$ cells with AFT024 feeders in the presence of Flt-3 ligand, stem cell factor (SCF), and interleukin-7 (IL-7) supports self-renewing cell divisions of primitive myeloid-lymphoid initiating cells. In concordance with that, Dr. Verfaillie provided evidence to demonstrate that CD34 ${ }^{1}$ cells cultured under these cultures for up to 4 weeks continued to have non-obese diabetic (NOD) SCID repopulating ability [6].

The question, which of these models most reliably measures HSC frequency and potential, remains unanswered. It can be argued that competitive repopulation assays, commonly used to measure murine HSC, but not presented by any of the speakers, is the most stringent measure of HSC [7]. How the requirement of human HSC to home to and engraft in a xenogeneic microenvironment impacts on HSC measurement is not known. The latter problem is obviously not encountered in the syngeneic Rhesus-Macaque transplantation model. If and how single cells, that have in vitro self renewal and multilineage differentiation potential compare with the engrafting HSC still needs to be further explored.

\section{Stem cell expansion and homing}

Engraftment requires that cells "home" to the marrow microenvironment. This was discussed by Drs. M. Moore and Quesenberry. Both speakers used in vitro model systems to assess adhesion and migration of hematopoietic progenitors and correlated the in vitro observations with in vivo homing/engraftment. Dr. Moore demonstrated that the chemokine receptor, CXCR4, plays a major role in allowing migration of hematopoietic cells to stromal feeders that support hematopoiesis in vitro [8]. Additional studies in which hematopoietic cells were cultured short- or long-term with high concentrations of cytokines indicated that migration diminishes depending on the duration of cytokine exposure and on the type of cytokine used. Decreased migration may underlay the decreased engraftment seen for progenitors cultured ex vivo prior to transplantation. Similar results were seen by Dr. Quesenberry, who showed that changes in cell cycle phase of HSC induced by ex vivo exposure to cy- tokines may in part explain decreased engraftment seen in vivo [9]. He provided further suggestive evidence that changes in adhesion receptor expression (VLA4, VLA5, PECAM, L-selectin) and function (adhesion to stroma) correlate with decreased engraftment [10]. These findings prompted both speakers to propose that engrafting ability of an ex vivo expanded stem cell population, will not only need to take into account the number of primitive progenitors present in the cell population but also the percent of these cells that can migrate/adhere.

\section{The potential impact of genomics}

The major drawback of the in vivo or in vitro models presented is their cumbersome nature and the technical expertise required. Further, these assays will not be clinically useful to monitor ex vivo manipulated populations for stem cell content. One solution is to identify a molecular "signature" of long-term repopulating HSC and their more committed progeny, which will allow enumeration of HSC present in populations from different origin or after ex vivo manipulation without requiring functional characterization discussed above (i.e., engraftment, multilineage potential). This was addressed by Drs. Lemischka, K. Moore, M. Moore, and Emerson.

Dr. Lemischka provided updated information on the stem cell gene database that he and other investigators are developing. The database now contains over 13,000 sequences derived from subtraction screens of murine fetal liver HSC, murine adult BM HSC, and human CD34 ${ }^{1}$ $\mathrm{CD} 38^{2}$ and $\mathrm{CD} 34^{2} \mathrm{Lin}^{2}$ cells. The database will contain over 40,000 sequences by the year 2000 . The stem cell database connects to EST, STS, Swiss protein, and Unigene databases; and provides virtual Northern, nearest neighbor, and library statistics. Dr. Lemischka used genes expressed exclusively in murine fetal liver hemopoietic stem cells but not more mature cells as an example. More than $70 \%$ of the sequences represent known genes, of which more than $50 \%$ encode for cell signaling molecules. Several of the novel sequences are homologous to genes characterized in Drosophila to be important in early development indicating that the database represents genes important in stem cell maintenance and/or differentiation. Gene micro-arrays will shortly be available to screen presumed HSC populations for their "stemness."

Dr. K. Moore has characterized more than 2000 known and novel genes expressed in the AFT024 feeder, known to support long-term repopulating murine as well as human HSC ex vivo, but not in feeders that do not support longterm repopulating cells. This complement of genes is also part of the stem cell gene database. As is true for genes from HSC, more than $50 \%$ of the genes identified in the subtraction screen between AFT024 and nonsupportive feeders are known signal transduction, structure, or transmembrane proteins, while approximately $40 \%$ of the genes are novel. 
Micro-arrays are being constructed that can be used to screen feeder layers for the presence or absence of genes thought to be responsible for the HSC supportive nature of the feeder.

\section{Candidate stem cell self-renewal genes}

Drs. M. Moore and Emerson then discussed two gene families thought to play an important role in stem cell biology, telomerase, and homeobox genes. Genes in the Hox family of homeobox genes play an important role in regulation of proliferation and differentiation processes in hematopoiesis [11]. What regulates expression of these genes is not known. Dr. Emerson discussed studies from his laboratory evaluating cis-acting elements that may regulate Hox-B4 in HSC. He identified a sequence, termed USS1, which inactivates Hox-B4 expression. Studies are currently ongoing to identify how USS1 itself is regulated as this may help decipher mechanisms underlying maintenance of stem cell phenotypes. Dr. Moore described the loss in telomere length observed after ex vivo expansion of CD34 ${ }^{1}$ cells [12]. The potential impact such telomere shortening may have in the clinical setting was illustrated through data from a study done at the University of Arkansas. Drs. Moore and Barlogie found a very strong correlation between the dose of CD34 ${ }^{1}$ cells infused in multiple myeloma patients undergoing transplantation, telomere length of cells after transplantation, and development of myelodysplasia or AML. Thus, in vivo (as seen here in the setting of small cell doses used for transplantation) or in vitro expansion may compromise the longevity of HSC and genomic stability of HSC following transplantation. Whether introduction of the telomerase gene will alleviate this potential problem remains to be seen.

Speakers and workshop participants were enthusiastic about the progress made in characterizing stem cell specific genes. There is significant optimism that this field will progress in the next few years to the point that it will be possible to genetically identify different subpopulations of HSC and this independent of the place of origin, or the ex vivo or in vivo manipulation of the cell. This will, however, require very close collaboration between investigators skilled in the functional characterization and those involved in genetic characterization of HSC.

\section{Goals of ex vivo stem cell expansion}

In the last group of presentations, several participants discussed ex vivo expansion of HSC. A number of different questions were addressed:

What culture condition will allow expansion of HSC?

Is the long-term repopulating HSC the cell that needs to be expanded or do we need to concentrate on more committed cells that will allow earlier engraftment?
Do we also need to pay attention to the need for expanding accessory cells, such as $\mathrm{T}$ lymphocytes, dendritic cells or macrophages?

What are the regulatory issues for expansion of $\mathrm{PB}$ or BM HSC?

Dr. McNiece argued that rather than expanding longterm repopulating, HSC expansion of intermediate cells that can alleviate neutropenia in the setting of high dose therapy is needed. He presented data from two clinical trials in which PB or UCB CD $34^{1}$ cells expanded in serum-free medium, SCF, MGDF, and G-CSF were used for transplantation [13]. Studies done by Dr. Reiffers in Bordeaux, France, showed that infusion of ex vivo expanded autologous PB cells prevented neutropenia following high dose therapy in the majority of patients that received these cells without interfering with long-term engraftment. Studies done at the University of Colorado in which patients were transplanted with cord blood grafts that were partly ex vivo expanded showed that engraftment can be obtained with small numbers of CD $34^{1}$ cells and that the ex vivo expansion step may allow for earlier engraftment. Although these studies were done in a nonrandomized fashion and long-term follow-up is lacking, these studies provide encouraging data suggesting that ex vivo expansion may generate a large population of early engrafting cells to prevent neutropenia following transplant.

Dr. Torok-Storb postulated that it may not be necessary to expand HSC but that we should concentrate on the generation of the correct set of accessory cells that will aid in early engraftment and prevent relapse. To illustrate this, she presented data to indicate that macrophages present in mobilized peripheral blood mononuclear cells produce interleukin-10 (IL-10) that has tolerizing effect [14]. This may explain the exciting new findings from a randomized multicenter study that will be presented at the American Society of Hematology Meetings showing that allogeneic mobilized PB progenitors allow engraftment with less significant graft-versus-host disease. These results support the notion that macrophages induce tolerance and, therefore, less significant problems in an allotransplant setting. Thus, investigators attempting to ex vivo expand grafts will need to pay attention to the types of cells that are present in the graft after manipulation.

\section{Ex vivo culture conditions}

A number of speakers presented data on preclinical and clinical studies in which ex vivo expansion of HSC was attempted. As discussed above, Drs. Nolta, Zanjani, and Dunbar transplanted ex vivo expanded cells in their respective in vivo animal models. All showed a significant decline in engraftment of HSC when cells were expanded in the absence of stromal cells or the extracellular matrix component fibronectin. In contrast, Drs. Heimfeld, Bartelemez, and Verfaillie (see above) provided evidence to suggest that cul- 
ture in contact with stromal feeders or in medium conditioned by such feeders may maintain, and possibly, expand HSC. Dr. Heimfeld showed that supernatants generated by stromal feeders such as MS5 support ex vivo expansion of committed and primitive hemopoietic cells, mature megakaryocyte precursors, and lymphoid cells. When transplanted in the preimmune fetal sheep model, engraftment occurred in primary recipients and secondary recipients, although the HSC pool could be more easily exhausted than when unmanipulated cells were used [15]. Data was presented showing early engraftment in three patients transplanted with cells expanded in this manner.

Dr. Bartelemez [16] discussed studies from his group indicating that the addition of thrombopoietin alone to Dexter-type long-term murine bone marrow cultures allows for massive expansion of long-term repopulating HSC. The mechanism underlying this surprising observation is under examination. Whether this will hold true for human HSC is also being examined.

\section{Regulatory issues}

Dr. Stephens from the FDA addressed regulatory issues that will be involved in clinical scale routine ex vivo manipulation of HSC. This will impact significantly on the type of culture conditions used. A number of investigators are currently testing both defined conditions as well as media conditioned by certain stromal feeders that, in an animal model, allowed for maintenance/expansion of HSC. Ultimately, the FDA will required that components used in ex vivo HSC culture systems are characterized and purified to be added to defined culture conditions.

\section{Conclusions}

In conclusion, there was consensus that it will be necessary to expand populations of cells with different functions: long-term repopulating cells, more committed early repopulating cells, and accessory cells. The need for expanding one or the other cell populations will differ according to the stem cell population used for transplantation, as UCB may be deficient in both short-term and long-term repopulating cells, purified adult BM or PB cells may be deficient in committed cells and/or accessory immune modulatory cells. True advances in this field will require that we develop better insights in the phenotype of the long-term and short term repopulating stem cells. Although significant progress has been made in defining model systems to test hematopoietic stemness of cells, these assays are cumbersome and do not provide real-time information concerning the type of cells present in a graft. Only when we can enumerate and qualitatively define HSC in a graft will it be possible to truly address which cells are required in a graft for successful and early engraftment: long-term HSC, short-term cells, and ac- cessory macrophages and/or lymphocytes. Final proof that a graft with certain in vitro or in vivo preclinical characteristics can successfully reconstitute hematopoiesis will require collaborative efforts between labs versant in in vitro or in vivo modeling of hematopoiesis as well as investigators studying the genetic characteristics of HSC. Therefore, plans are being made to start to correlate genetic phenotype of HSC with their function in preclinical in vitro and in vivo models, and ultimately, in vivo transplantation in humans.

\section{References}

1. Nolta J, Dao M, Wells S, Smogorzewska E, Kohn D (1996) Transduction of pluripotent human hematopoietic stem cells demonstrated by clonal analysis after engraftment in immune-deficient mice. Proc Natl Acad Sci U S A 93:2414

2. Dunbar CE, Seidel NE, Doren S, Sellers S, Cline AP, Metzger ME, Agricola BA, Donahue RE, Bodine DM (1996) Improved retroviral gene transfer into murine and Rhesus peripheral blood or bone marrow repopulating cells primed in vivo with stem cell factor and granulocyte colony-stimulating factor. Proc Natl Acad Sci U S A 93:11871

3. Shimizu Y, Ogawa M, Kobayashi M, Almeida-Porada G, Zanjani ED (1998) Engraftment of cultured human hematopoietic cells in sheep. Blood 91:3688

4. Punzel M, Wissink SD, Miller JS, Moore KA, Lemischka IR, Verfaillie CM (1999) The myeloid-lymphoid initiating cell (ML-IC) assay assesses the fate of multipotent human progenitors in vitro. Blood 93:3750

5. Moore KA, Hideo E, Lemischka IR (1997) In vitro maintenance of highly purified transplantable hematopoietic stem cells. Blood 89:4337

6. Larochelle A, et al. (1995) Engraftment of immune-deficient mice with primitive hematopoietic cells from beta-thalassemia and sickle cell anemia patients: implications for evaluating human gene therapy protocols. Hum Mol Genet 4:163

7. Harrison DE, Zhong RK, Jordan CT, Lemischka IR, Astle CM (1997) Relative to adult marrow fetal liver repopulates nearly five times more effectively long-term than short-term. Exp Hematol 25:293

8. Möhle R, Bautz F, Rafii S, Moore MAS, Brugger W, Kanz L (1998) The chemokine receptor CXCR-4 is expressed on CD341 hematopoietic progenitors and leukemic cells and mediates transendothelial migration induced by stromal cell-derived factor-1. Blood 91:4523

9. Quesenberry P, et al. (1999) Stem cell engraftment and cell cycle phenotype. Leukemia 13:92

10. Becker P, Nilsson SK, Li Z, Berrios VM, Dooner MS, Cooper CL, Hsieh C, Quesenberry P (1999) Adhesion receptor expression by hematopoietic cell lines and murine progenitors: modulation by cytokines and cell cycle status. Exp Hematol 27:533

11. Thorsteinsdottir U, Sauvageau G, Humphries R (1997) Hox homeobox genes as regulators of normal and leukemic hematopoiesis. Hematol Oncol Clin North Am 11:1221

12. Yui J, Chiu C, Lansdorp P (1998) Telomerase activity in candidate stem cells from fetal liver and adult bone marrow. Blood 91:3255

13. Purdy MH, et al. (1995) Large volume ex vivo expansion of CD34positive hematopoietic progenitor cells for transplantation. J Hematother 4:515

14. Tanaka J, Imamura M, Kasai M, Asaka M, Torok-Storb B (1999) The role of accessory cells in allogeneic peripheral blood stem cell transplantation. Int J Hematol 69:70

15. Roecklein B, et al. (1997) Serial xenogeneic transplantation of ex vivo expanded human CD341 cells. Blood 90:1750a

16. Yagi M, Ritchie KA, Sitnicka E, Storey C, Roth GJ, Bartelmez S (1999) Sustained ex vivo expansion of hematopoietic stem cells mediated by thrombopoietin. Proc Natl Acad Sci U S A 96:8126 